\title{
Dataset for Scheduling Strategies for Microgrids Coupled with Natural Gas Networks
}

\author{
Muhammad Yousif ${ }^{1, *(\mathbb{D}}$, Qian Ai ${ }^{1, *}$, Yang Gao ${ }^{1}{ }^{\mathbb{D}}$, Waqas Ahmad Wattoo ${ }^{1,2}$, Ran Hao ${ }^{1}$ and \\ Ziqing Jiang ${ }^{1}$ \\ 1 Key Laboratory of Control of Power Transmission and Conversion (SJTU), Ministry of Education, \\ Shanghai 200240, China; jjgyxky@126.com (Y.G.); xs2waqas88@hotmail.com (W.A.W.); \\ haoransjtu@sjtu.edu.cn (R.H.); jiangzq_jollay@sjtu.edu.cn (Z.J.) \\ 2 Department of Electrical and Computer Engineering, COMSATS University Islamabad (CUI), \\ Sahiwal Campus, Punjab 57000, Pakistan \\ * $\quad$ Correspondence: yousif@sjtu.edu.cn (M.Y.); aiqian@sjtu.edu.cn (Q.A.); Tel.: +86-21-34204584 (Q.A.)
}

Received: 30 December 2018; Accepted: 2 February 2019; Published: 5 February 2019

\begin{abstract}
Datasets are significant for researchers to test the functionality of their proposed strategies for the microgrid dispatch. This article presents a dataset to help researchers in testing their algorithms related to the dispatch problem of microgrids coupled with natural gas networks. This preliminary release of a microgrid dispatch dataset contains data related to microgrid components (like solar PV, wind turbine, fuel cell and batteries) and natural gas network elements connected with the microgrid (e.g., micro gas turbine). It also includes the data associated with the authors' proposed scheduling strategy and its dispatch results. The provided dataset can be used to reproduce the authors' proposed strategy. The presented dataset further can be used for comparisons of other researchers' proposed strategies. These comparisons will make a strategy's features more evident.
\end{abstract}

Dataset: Available as the supplementary file. Matlab Code and Dataset for Scheduling Strategies for Microgrids (www.mdpi.com/2306-5729/4/1/24/s1).

\section{Dataset License: CC-BY}

Keywords: power scheduling strategy data; distribution networks; renewable energy sources; optimal power dispatch; dataset

\section{Summary}

The primary energy networks are transforming from centralized to distributed, and developing into more interconnected networks [1,2], for example, the electricity grid and natural gas network. Furthermore, these two networks have started coupling to support each other and overcome their problems. A micro gas turbine (MT) works as a coupling gear between these two energy networks. Nowadays, distributed electricity grids have more different types of energy sources (including MT), which make the electric power dispatch more challenging [3,4]. Many researchers focused on this dispatch problem and proposed a number of new dispatch strategies. Datasets play a significant role in measuring efficiency and accuracy of a microgrid. Testing and comparing a proposed strategy are crucial parts of the research process. Most researchers claimed that their proposed dispatch strategy is the most efficient one. Some of these claims are based on a vague comparison. Most of the researchers did not provide such a piece of detailed information related to their claims, which are essential to verify and compare the strategy with other proposed strategies. Here, the significance of a commonly accessible dataset arises so that one can test its dispatch algorithm in the same transparent 
conditions. This will make their algorithms' comparison more evident and significant. In this data descriptor article, the authors provided the dataset and mathematical models related to different components of microgrids, and a proposed strategy's results. The dataset, mathematical models and proposed strategy's results can be useful in multiple ways. The dataset can be used in testing and measuring other dispatch algorithms' efficiency and accuracy. The models can be used in preparing new algorithms.

The aforesaid dataset contains hourly readings of metrological information for renewable energy sources (RESs), microgrid components' mathematical models and the proposed strategy's results.

\section{Data Description}

This dataset (supplementary file) contains hourly power produced by PV, a wind turbine, micro gas turbine and fuel cell. It also contains these power generation sources' mathematical models, which can be used to calculate the power produced according to provided input information. The description of the provided files is given in Table 1.

Table 1. Dataset's file names and descriptions.

\begin{tabular}{cc}
\hline File Name & Description \\
\hline Basic_input.m & Basic input about metrological and price info \\
Bat.m & Battery's function \\
Cost_calculator.m & Microgrids' one-day cost calculator \\
Dispatch_all.mat & Hourly power flow from all microgrid players \\
FuelCell.m & Fuel cell output power and cost calculation \\
Gas_dispatch.m & Natural gas dispatch for microturbines \\
max60.m & Current generated by solar PV \\
micro_gas_turbine.m & Micro gas turbine power and cost calculation \\
MicroGrid_Dispatch.m & The main function for power dispatch \\
PandO.m & Solar PV maximum power point tracking \\
Pwt.m & Power generation calculation by wind turbine \\
Micro_grid_parameter.m & Microgrid's DER and load parameters \\
\hline
\end{tabular}

The file's content is given at the start of each file. Some of these files are about the basic input values, which are further used by microgrids' components models. These components' models are able to calculate the respective power curves and cost curves.

\section{Methods}

MATLAB software is used in preparing the dataset. All the microgrid's components' models are coded in MATLAB. The presented models and their coefficients are based on $[5,6]$, which further refer to [7-9]. Furthermore, the Fuel Cell model is deduced from [10] and the Micro Turbine model is deduced from [11,12]. The pollution treatment cost and other cost-related equations and coefficients are based on [13,14]. The authors proposed a dispatch strategy in their previous study [3]. The final result of the authors' proposed strategy is also given in the MATLAB data format in file "Dispatch_all.mat" (Figure 1). In this data file, the 24-hour dispatch solution and power flow from all the components of the microgrid are given. This dispatch solution is calculated as per the input information related to electric grids and natural gas network, which is also provided in the MATLAB file named "Basic_input.m".

The components' models related to the microgrid are coded as MATLAB functions so that they can be reused or integrated easily. The PV-related model also contains the MPPT algorithm which can be replaced by a preferred technique. The approach to code the components' models as MATLAB functions will result in enhanced readability and reusability of the dataset. 


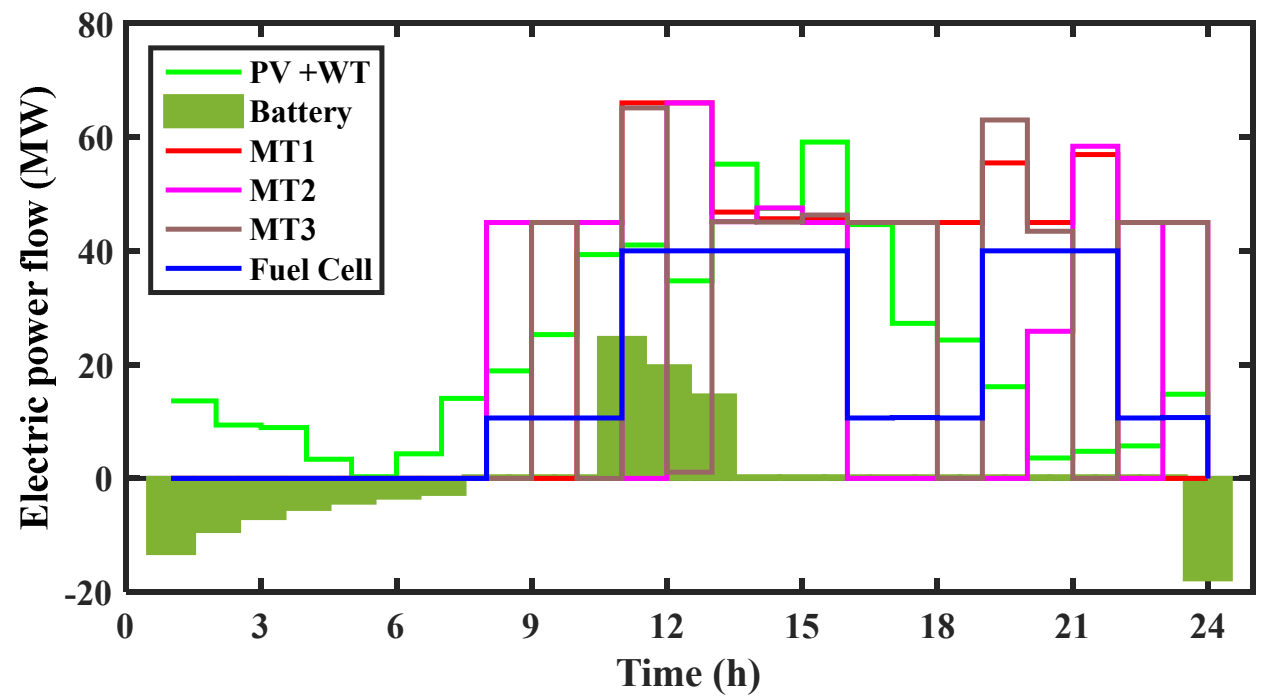

Figure 1. Graphical representation of the data in file "Dispatch_all.mat".

\section{User Notes}

Three different sets of hourly wind speeds are provided in the file "Basic_input.m". Each set of these wind speeds corresponds to the seasonal pattern. The accuracy of these wind speed sets is up to 2 decimal points. Along with wind speed data, three sets of solar radiations corresponding to the seasonal pattern are also present in the file. The main file of the coded models is "MicroGrid_Dispatch.m" which calls (is linked with) other models' functions and also contains the code related to plotting graph results.

\section{Limitations and Recommendations}

Along with the dataset, this article also presents two more things; the mathematical models and the results of the authors' proposed strategy (available as the supplementary file). As all of these things are coded in MATLAB, direct use of the dataset is limited to MATLAB software. However, one can export the dataset into other-software-readable formats, as MATLAB has supported the exporting of data in a number of different formats. One can substitute any of these offered parts of the dataset and compare its results. This will not only make the comparison easier, but also make the implementation of a new technique easier due to provided coded models.

Supplementary Materials: Matlab Code and Dataset for Scheduling Strategies for Microgrids is available online at http:/ / www.mdpi.com/2306-5729/4/1/24/s1.

Author Contributions: Conceptualization and methodology, M.Y. and Q.A.; software, and data curation, M.Y. and Y.G; writing—original draft preparation, M.Y.; writing—review and editing, M.Y., W.A.W., R.H. and Z.J.; supervision and funding acquisition, Q.A.

Funding: This work was supported by National Natural Science Foundation of China (U1866206).

Conflicts of Interest: The authors declare no conflict of interest.

\section{Abbreviations}

RES Renewable Energy Source

DER Distributed Energy Source

FC Fuel cell

MPPT Maximum Power Point Tracking

PV Photovoltaic

MATLAB MATrix LABoratory software developed by MathWorks 


\section{References}

1. Qian, A.; Ran, H. Key technologies and challenges for multi-energy complementarity and optimization of integrated energy system. Autom. Electr. Power Syst. 2018, 42, 2-10.

2. Yousif, M.; Ai, Q.; Wattoo, W.A.; Jiang, Z.; Hao, R.; Gao, Y. Least cost combinations of solar power, wind power, and energy storage system for powering large-scale grid. J. Power Sources 2019, 412, 710-716. [CrossRef]

3. Yousif, M.; Ai, Q.; Gao, Y.; Wattoo, W.A.; Jiang, Z.; Hao, R. Application of Particle Swarm Optimization to a Scheduling Strategy for Microgrids Coupled with Natural Gas Networks. Energies 2018, 11, 3499. [CrossRef]

4. Yousif, M.; Ai, Q.; Gao, Y.; Wattoo, W.A.; Jiang, Z.; Hao, R. An Optimal Dispatch Strategy for Distributed Micro-Grids. In Proceedings of the 2018 2nd IEEE Conference on Energy Internet and Energy System Integration (EI2), Beijing, China, 20-22 October 2018; pp. 1-5.

5. Kou, Y.N.; Zheng, J.H.; Li, Z.; Wu, Q.H. Many-objective optimization for coordinated operation of integrated electricity and gas network. J. Mod. Power Syst. Clean Energy 2017, 5, 350-363. [CrossRef]

6. Perera, A.T.D.; Nik, V.M.; Mauree, D.; Scartezzini, J.L. Electrical hubs: An effective way to integrate non-dispatchable renewable energy sources with minimum impact to the grid. Appl. Energy 2017, 190, 232-248. [CrossRef]

7. Jing, Z.X.; Jiang, X.S.; Wu, Q.H.; Tang, W.H.; Hua, B. Modelling and optimal operation of a small-scale integrated energy based district heating and cooling system. Energy 2014, 73, 399-415. [CrossRef]

8. An, S.; Li, Q.; Gedra, T.W. Natural gas and electricity optimal power flow. In Proceedings of the 2003 IEEE PES Transmission and Distribution Conference and Exposition (IEEE Cat. No.03CH37495), Dallas, TX, USA, 7-12 September 2003; Volume 1, pp. 138-143. [CrossRef]

9. Martinez-Mares, A.; Fuerte-Esquivel, C.R. A unified gas and power flow analysis in natural gas and electricity coupled networks. IEEE Trans. Power Syst. 2012, 27, 2156-2166. [CrossRef]

10. Barbir, F.; Gómez, T. Efficiency and economics of proton exchange membrane (PEM) fuels cells. Int. J. Hydrogen Energy 1996, 21, 891-901. [CrossRef]

11. Abido, M.A. Environmental/Economic Power Dispatch Using Multiobjective Evolutionary Algorithms. IEEE Trans. Power Syst. 2003, 18, 1529-1537. [CrossRef]

12. Campanari, S.; Macchi, E. Technical and Tariff Scenarios Effect on Microturbine Trigenerative Applications. In Proceedings of the ASME Turbo Expo 2003, Collocated with the 2003 International Joint Power Generation Conference, Atlanta, GA, USA, 16-19 June 2003; pp. 747-757.

13. Krishnamurthy, S.; Tzoneva, R. Impact of price penalty factors on the solution of the combined economic emission dispatch problem using cubic criterion functions. In Proceedings of the 2012 IEEE Power and Energy Society General Meeting, San Diego, CA, USA, 22-26 July 2012.

14. Kefayat, M.; Lashkar Ara, A.; Nabavi Niaki, S.A. A hybrid of ant colony optimization and artificial bee colony algorithm for probabilistic optimal placement and sizing of distributed energy resources. Energy Convers. Manag. 2015, 92, 149-161. [CrossRef]

(C) 2019 by the authors. Licensee MDPI, Basel, Switzerland. This article is an open access article distributed under the terms and conditions of the Creative Commons Attribution (CC BY) license (http://creativecommons.org/licenses/by/4.0/). 